\title{
Characterization of a Severe Strain of Cucumber Mosaic Cucumovirus Seedborne in Cowpea
}

A. G. Gillaspie, Jr., USDA, ARS, Plant Genetic Resources Conservation Unit, Griffin, GA 30223-1797; and M. R. Hajimorad and S. A. Ghabrial, Plant Pathology Department, University of Kentucky, Lexington 40546

\begin{abstract}
Gillaspie, A. G., Jr., Hajimorad, M. R., and Ghabrial, S. A. 1998. Characterization of a seedborne strain of cucumber mosaic cucumovirus from cowpea. Plant Dis. 82:419-422.

A new seedborne strain of cucumber mosaic cucumovirus (CMV) that induces severe symptoms on many cowpea genotypes was detected in Georgia in 1994. This strain, designated CMV-Csb, is asymptomatic on tobacco, but it produces more severe cowpea stunt symptoms when present in combination with blackeye cowpea mosaic potyvirus than do the more prevalent CMV isolates. The new strain is seedborne in cowpea (1.5 to 37\%), has no associated satellite RNA, and is classified as a member of subgroup I of CMV strains based on nucleic acid hybridization assays.
\end{abstract}

In 1994, a severe viruslike disease with unusual symptoms was observed in cowpeas (Vigna unguiculata (L.) Walp. subsp. unguiculata) in a germ plasm regeneration field near Griffin, Georgia. The symptoms were comprised of severely distorted leaves with a prominent green mosaic on a nearly white background. Symptom severity varied somewhat depending upon the cowpea line involved. Serological tests (enzyme-linked immunosorbent assay [ELISA]) of field-collected plants indicated the presence of cucumber mosaic cucumovirus (CMV), cowpea severe mosaic comovirus (CSMV), and southern bean mosaic sobemovirus (SBMV). Furthermore, samples that tested positive for CMV also reacted with an antiserum to peanut stunt cucumovirus (PSV) from Clemson University, but not with PSV antisera from the University of Kentucky. The serological tests, therefore, did not provide conclusive results as to the identity of the virus (or virus complex) responsible for the unusual symptoms. In 1995, a similar virus problem was observed in a field of mung beans (Vigna radiata (L.) R. Wilczek var. radiata) in the same region near Griffin.

During the course of separating the virus mixture to obtain cultures of the individual viruses involved, we isolated and partially characterized a new seedborne strain of CMV that induces unusually severe

Corresponding author: A. G. Gillaspie, Jr.

E-mail: s9gg@ars-grin.gov

Accepted for publication 7 January 1998.

Publication no. D-1998-0213-01R

This article is in the public domain and not copyrightable. It may be freely reprinted with customary crediting of the source. The American Phytopathological Society, 1998. symptoms in cowpea, designated CMVCsb. In this report, we describe some biological, serological, and biochemical properties of CMV-Csb. Because of the initial confusion regarding the identity of the cucumovirus isolates (CMV or PSV), we also describe some biological and biochemical properties of a PSV isolate from peanut for comparison purposes. We concluded, based on the biological properties and nucleic acid hybridization assays, that CMV-Csb was the causal agent of the unusually severe disease of cowpeas observed in Georgia and that it is a member of subgroup I of CMV strains.

\section{MATERIALS AND METHODS}

Viruses. Initial studies were made with virus isolates from field-grown cowpeas with apparent mixed virus infections (CMV/PSV, SBMV, and CSMV). In order to obtain a culture of CMV free from SBMV and CSMV, leaf extracts were prepared from infected cowpeas showing the unusual severe symptoms and mechanically inoculated onto tobacco (Nicotiana tabacum L. cv. Burley 21) to eliminate SBMV. CSMV was eliminated through seed transmission in cowpea by testing for those seedlings that contained no CSMV. CSMV is seedborne in cowpea at a rate of $10 \%$ or less.

Subcultures of tomato aspermy virus (TAV) strain V (5) and CMV strains Fny (CMV subgroup I) and LS (CMV subgroup II) (14) were provided by K. L. Perry (Purdue University, West Lafayette, IN) and M. J. Roossinck (Noble Foundation, Ardmore, OK), respectively. PSV strains ER (subgroup I) and W (subgroup II) have been previously described (7). An isolate of PSV from peanuts in Georgia (PSV-peanut) was also used in some experiments. All viruses were maintained and propagated in Burley tobacco cv. Ky 14.
CMV, PSV, and TAV virions were purified for use in some experiments.

Seed transmission and serology. Seeds were planted in plastic trays in Metro-Mix 300 (Grace Sierra Horticultural Products Co., Milpitas, CA) and grown in an insectfree greenhouse. Seedlings were sampled when the plants reached the third trifoliolate stage. Leaf samples were triturated at a $1: 10$ dilution (wt/vol) in extraction buffer, and 200- $\mu \mathrm{l}$ aliquots were dispensed into two replicate microtiter plate wells (flatbottom Immulon II plates, Dynatech Laboratories Inc., Chantilly, VA) for each of the virus-specific direct antigen coating (DAC)-ELISA plates (4). Buffers and procedures were previously described (4). Viral identification assays were done on individual plants of about the same age and using the same ELISA procedures. The sources of the polyclonal antisera used were (dilutions indicated in parentheses): PVAS-470 CSMV (1:10,000), American Type Culture Collection, Rockville, Maryland; cowpea chlorotic mottle bromovirus (CCMV) (1:5,000), CMV (1:5,000), PSV $(1: 20,000)$, and SBMV $(1: 20,000)$, O. W. Barnett, Clemson University, Clemson, South Carolina; and blackeye cowpea mosaic potyvirus (BlCMV) (1:20,000), J. W. Demski, University of Georgia, Griffin. The University of Kentucky antisera to the two subgroups of PSV strains $(1: 18,000)$ were previously described (7).

Host range. Host range studies were done in an insect-free greenhouse on symptomatic plant samples directly from the field and on a culture of CMV-Csb freed from other viruses as previously described. PSV-peanut was used for comparison in these tests. Plant species used were cowpea PIs 270068, 292899, 302458, and 307561; Chenopodium album subsp. amaranticolor Coste \& A. Reyn.; tobacco cv. Burley 21; and Phaseolus vulgaris L. cv. Top Crop. All plants were inoculated mechanically by rubbing Carborundum-dusted leaves with plant extracts in $0.025 \mathrm{M}$ phosphate buffer, $\mathrm{pH}$ 7. Plants were observed for symptoms over a period of 15 to 25 days.

The ability of CMV-Csb to interact synergistically with BlCMV and elicit the cowpea stunt disease in doubly-infected plants (15) was tested in cowpea cv. Coronet. An isolate of BlCMV was provided by J. W. Demski. As a positive control, a mixed inoculum of BlCMV and CMVlupine (J. W. Demski) was inoculated onto 
healthy Coronet cowpea plants. Inocula of the individual viruses were also included. In a similar manner, an attempt was made to compare the symptoms induced in cowpea lines PI 292899, 302458, and 307561 by a mixed inoculum of CMV-Csb, CSMV, and SBMV with those elicited by CMVCsb alone.

dsRNA patterns. Leaf samples from the field as well as those from seed transmission studies were processed using both the standard extraction and the minibatch methods and analyzed for the presence of dsRNA, as described by Jordan and Dodds (8). The dsRNA fractions were analyzed by agarose-gel electrophoresis, and the gels were stained with ethidium bromide.

Viral RNA isolation and analysis. Viral RNAs were purified from virions by three phenol-chloroform extractions in the presence of $0.2 \mathrm{M}$ Tris buffer, $\mathrm{pH} 8.5$, containing $1 \mathrm{M} \mathrm{NaCl}, 1 \%$ sodium dodecyl sulfate (SDS), and $2 \mathrm{mM}$ EDTA. After concentration by ethanol precipitation, the RNA was resuspended in TE buffer (10 $\mathrm{mM}$ Tris- $\mathrm{HCl}$ and $1 \mathrm{mM}$ EDTA, $\mathrm{pH} 8.3$ ) and stored at $-20^{\circ} \mathrm{C}$. The RNAs were analyzed in a nondenaturing $1.8 \%$ agarose gel in the presence of TAE $(40 \mathrm{mM}$ Tris- $\mathrm{HCl}$, $\mathrm{pH} 8.0,20 \mathrm{mM}$ sodium acetate, and $2 \mathrm{mM}$ sodium EDTA) as previously described (6).

Synthesis of ${ }^{32} \mathbf{P}$-labeled cDNA probes. For slot blot hybridization, cDNA probes to viral RNAs from CMV-Fny, CMV-LS, PSV-ER, PSV-W, and TAV-V virions were prepared as previously described by Hajimorad et al. (6), except that Superscript reverse transcriptase (Gibco-BRL, Gaithersburg, MD) was used.

For Northern blot hybridization, cloned cDNA probes to CMV-Fny RNAs were used. These were prepared by nick translation (18) of the plasmids pFny 86.6 (17), pFny 209, and pFny 309 (16) containing full-length cDNA clones of Fny-CMV

Table 1. Comparative host range of strain Csb of cucumber mosaic virus (CMV-Csb) and the peanut isolate of peanut stunt virus (PSV-peanut) ${ }^{\mathrm{a}}$

\begin{tabular}{|c|c|c|}
\hline Host & CMV-Csb & PSV-peanut \\
\hline \multicolumn{3}{|l|}{ Vigna unguiculata } \\
\hline PI 270068 & ModMo, $\mathrm{Ma}^{\mathrm{b}}$ & Rdi/ MilMo \\
\hline PI 292899 & ChlS/ MilMo & ChlS/ MilMo \\
\hline PI 302458 & ChlS/ ModMo & ChlS/ MilMo \\
\hline PI 307561 & ChlS/ Mod-SevMo, Ma, VC & ChlS/ MilMo \\
\hline $\begin{array}{l}\text { Chenopodium album } \\
\text { subsp. amaranticolor }\end{array}$ & Llc & Llc \\
\hline $\begin{array}{l}\text { Phaseolus vulgaris } \\
\text { cv. Top Crop }\end{array}$ & ChlS, Rdi/ MilMo, VN & ChlS, Rdi \\
\hline $\begin{array}{l}\text { Nicotiana tabacum } \\
\text { cv. Burley } 21\end{array}$ & $\ldots^{\mathrm{c}}$ & $\mathrm{RSc} / \mathrm{VB}$ \\
\hline
\end{tabular}

${ }^{a}$ Isolates of CMV-Csb were obtained by passage of virus through one seedborne phase selecting for those isolates without cowpea severe mosaic comovirus followed by passage through tobacco to eliminate southern bean mosaic sobemovirus (SBMV) before inoculation of cowpeas; the peanut isolate of PSV (PSV-peanut) was obtained after passage through tobacco to eliminate possible coinfection with SBMV before inoculation of cowpea.

${ }^{\mathrm{b}}$ Virus-induced symptoms: $\mathrm{ChlS}=$ chlorotic spots; $\mathrm{Llc}=$ chlorotic local lesions; $\mathrm{Ma}=$ malformation (distortion, crinkle, etc.); Mil = mild; Mo = mottle or mosaic; Mod $=$ moderate; Rdi $=$ reddening of inoculated leaves; $\mathrm{Rsc}=$ chlorotic ringspot; $\mathrm{Sev}=$ severe $\mathrm{VB}=$ veinbanding or oak leaf pattern; $\mathrm{VC}=$ veinclearing; $\mathrm{VN}=$ vein necrosis

${ }^{\mathrm{c}}$ Symptomless plants tested positive for CMV by direct antigen coating (DAC)-ELISA. strains (7) did not react with the samples identified as CMV. The Kentucky PSV antiserum to subgroup I strains reacted strongly with the PSV-peanut isolate. Apparently, the PSV-Clemson antiserum contained antibodies directed to epitopes common for both CMV and PSV. Similar serological results (detection of CMV, CSMV, and/or SBMV) were obtained with field isolates inoculated to cowpeas in the greenhouse and with plants from seeds collected from infected plants in the field. No viruslike particles were detected by electron microscopy of dip preparations of crude leaf extracts. This suggests that no rod-shaped viruses were involved but did not rule out small viruses with spherical particles, since these were unlikely to be visualized in such crude preparations. Polyhedral particles of a size consistent with that of cucumoviruses, however, were observed by electron microscopy following clarification and concentration (by $8 \%$ polyethylene glycol (PEG) precipitation, low-speed centrifugation at $8,500 \times g$, and ultracentrifugation at $104,000 \times g$ ) of extracts from plants mechanically inoculated with field isolates.

Because the severe symptoms observed in some cowpea lines somewhat resembled those induced by geminiviruses, the possibility of a geminivirus coinfecting these cowpea plants was tested. This possibility, however, was ruled out (data not shown) using a geminivirus-specific polymerase chain reaction (PCR)-based assay (10). Search for diagnostic inclusion bodies in infected tissues revealed the presence of cucumoviruslike inclusion bodies, but no inclusions diagnostic of other virus families or genera were detected (data not shown).

After two passages through tobacco, CMV-Csb produced very mild symptoms on cowpea plants cv. Early Ramshorn. However, after a number of passages through cowpea plants, the more severe symptoms were again present on some cowpea lines even though these plants still did not contain CSMV or SBMV. This is reminiscent of the phenomenon of reversible host adaptation in CMV reported earlier by Yarwood (19).

The host range data for CMV-Csb are presented in Table 1. The cowpea genotypes responded in much the same way as in the field. The CMV-Csb isolate was symptomless in tobacco, although the virus did multiply in this host. CMV isolated from legumes typically produces symptoms in many lines of cowpeas (a moderate green-on-green mosaic), as well as in nonlegumes such as mosaic symptoms in tobacco (11). The PSV isolate from peanuts produced different symptoms than CMVCsb on tobacco, cowpeas, and beans (Table 1). Even the PSV-induced chlorotic local lesions on Chenopodium were broader and less well defined than those elicited by CMV-Csb. Interestingly, the symptoms 
produced in the host plant species tested following inoculation with the field isolates were very similar to those induced by CMV-Csb alone. Therefore, the data presented in Table 1 include only the host reactions to CMV-Csb alone. When a mixed inoculum of CMV-Csb, CSMV, and SBMV was applied to the same cowpea lines on which the disease was originally observed, the resultant symptoms were much more severe than those observed in the field, with more reddening and necrosis of inoculated leaves as well as the growing tips. It was thus concluded that CMV-Csb alone is sufficient to induce the original unusually severe symptoms observed in some cowpea lines and that mixed infections with SBMV and/or CSMV may lead to even more severe symptoms.

When CMV-Csb was inoculated onto a susceptible cowpea cultivar in combination with BlCMV, the cowpea stunt symptoms produced were much more severe than those induced by either virus alone. The symptoms on Coronet cowpea were stunting with severe reddening of trifoliolate leaves and petioles, mosaic, and necrosis. The symptoms of CMV-lupine plus BlCMV were also synergistic, as has been reported for cowpea stunt (15), but these symptoms were less severe than those produced with CMV-Csb plus BlCMV. These findings underscore the potential epidemiological significance of CMV-Csb in the development of cowpea stunt syndrome and similar serious synergistic disease syndromes. This is a particular concern since CMV-Csb is symptomless in some of its host species.
Results of seed transmission experiments showed that CMV-Csb was seedborne in cowpeas to the extent of 1.5 to $18.2 \%$ among three PIs and $37.6 \%$ in cv. Early Ramshorn. The rate of transmission varied with the cowpea genotype used. The PSV-peanut isolate was inoculated onto cowpea cv. Early Ramshorn and was not seedborne in the 460 seeds tested. This is in agreement with reports that PSV is not seedborne in cowpea (12). CMV was demonstrated to be seed transmitted in wild cucumber (3). A number of reports deal with seed transmission of CMV in cowpeas. Anderson (1) found seed transmission of CMV in cowpea cultivars (4 to 28\%), and Meiners et al. (11) observed up to $30 \%$ seed transmission of a Puerto Rican strain of CMV in cowpeas, depending upon the genotype tested. In evaluations of cowpea germ plasm collections, Bashir and Hampton (2) reported on seedborne CMV at levels of 0 to $2 \%$. These reports describing CMV seed transmission in cowpeas characterized the virus based upon host range, serology, physical properties in sap, electron microscopy, and insect transmission.

The dsRNA profiles obtained with isolates of CMV-Csb and PSV-peanut were consistent with cucumovirus-sized genomic RNAs. Furthermore, bands corresponding to the dsRNA forms of satellite RNA (13) were detected with PSV-peanut, but not with CMV-Csb (data not shown). To ensure that PSV was not coinfecting the cowpea plants, reverse transcription (RT)PCR using primers specific for PSV RNAs 2 and 3 (7) was performed, and the results

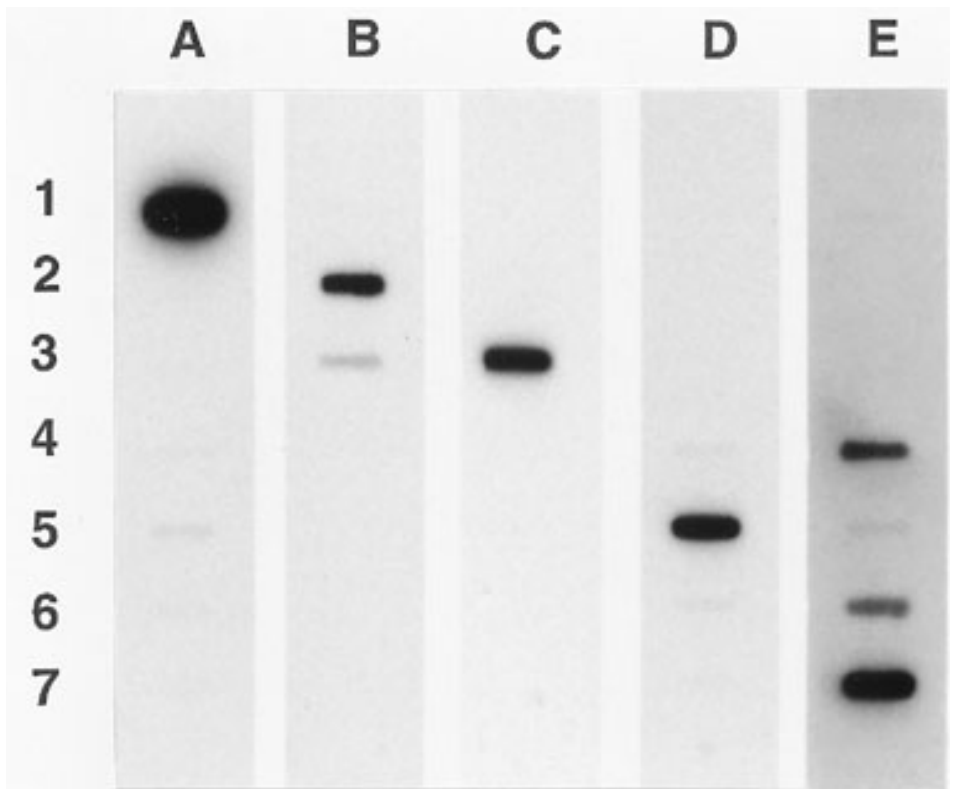

Fig. 1. Slot blot hybridization analysis of cucumovirus RNAs. Viral RNAs $(0.5 \mu \mathrm{g})$ in TE buffer isolated from purified virions of cucumber mosaic cucumovirus (CMV)-Fny (1), CMV-Csb isolate 276 (2), CMV-LS (3), CMV-Csb isolate 315 (4), peanut stunt cucumovirus (PSV)-ER (5), PSV-W (6) and tomato aspermy virus (TAV)-V (7) were spotted onto Hybond $\mathrm{N}^{+}$membranes and hybridized to ${ }^{32} \mathrm{P}$-labeled cDNA to genomic RNAs of CMV-Fny, CMV-LS, PSV-ER, PSV-W, and TAV-V, panels A to E, respectively. CMV-Csb isolates hybridized only with cDNA probes to CMV-Fny RNAs.

were negative for PSV (data not shown). Conclusive evidence for the presence of CMV, and not PSV, in these plants was obtained with the slot blot and Northern hybridization experiments. In slot blot hybridization assays (Fig. 1) using cDNA probes specific to CMV subgroups I and II (panels A and B, respectively), PSV subgroups I and II (panels $\mathrm{C}$ and $\mathrm{D}$, respectively), and TAV (panel E), strong hybridization signals were detected only when CMV-Csb isolates were reacted with a CMV subgroup I cDNA probe. Very weak or no hybridization was observed when the CMVCsb isolates were reacted with the other four probes, even though the latter hybridized strongly with their homologous RNAs (Fig. 1 ). These results were further confirmed by Northern hybridization assays using cloned cDNA probes to CMV-Fny RNAs 1, 2, and 3, a subgroup I strain (Fig. 2).

In conclusion, the implications of this severe seedborne strain of CMV for germ plasm handling and for growers are potentially highly significant. The tendency is for germ plasm workers to overlook common strains of CMV, since the virus is found in all growing areas of the world and there are no known sources of resistance in Vigna spp. This severe strain could be moved to other areas with Vigna germ plasm shipments in genotypes in which the virus produces mild symptoms. If it were widely introduced into growing areas, growers could face more significant losses from cowpea stunt. One solution would be to limit the movement of any CMV-infected germ plasm. We have no evidence of the original source of this strain in Georgia or how long it was present before detection. CMV has the potential to remain in a region for long periods, even in the absence of crops, because of its wide host range of perennial weeds.

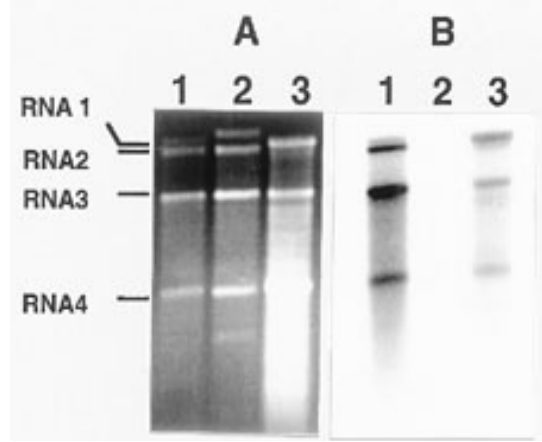

Fig. 2. Northern blot hybridization of cucumber mosaic cucumovirus (CMV) RNAs. RNAs isolated from virions of CMV-Fny (lanes 1), CMVLS (lanes 2), and CMV-Csb isolate 315 (lanes 3) were electrophoresed in $1.8 \%$ agarose gel and stained with ethidium bromide (panel A) or blotted onto a Hybond $\mathrm{N}^{+}$membrane and hybridized with ${ }^{32} \mathrm{P}-$-labeled cloned cDNA probes to FnyCMV genomic RNAs (panel B). The positions of RNAs 1 to 4 are indicated to the left. CMV-Csb is classified as a CMV subgroup I strain since it hybridized with cloned probes to CMV-Fmy RNAs, a known subgroup I strain of CMV. 


\section{ACKNOWLEDGMENTS}

We thank Chung Chi Hu for assistance with RTPCR analysis for PSV and dsRNA banding profiles, Jane Polston for her help with geminivirus PCR testing, Robert McGovern for examining tissue for the presence of viral inclusion bodies, Margaret Brannigan for her assistance with electron microscopy, and James Chalkley, Mark Hopkins, and David Pinnow for their technical assistance and observations during the seed transmission, host range, and serological tests. Mention of a trademark or a proprietary product does not constitute a guarantee or warranty of the product by the U.S. Department of Agriculture and does not imply its approval to the exclusion of other products that may also be available.

\section{LITERATURE CITED}

1. Anderson, C. W. 1957. Seed transmission of three viruses in cowpea. Phytopathology 47:515.

2. Bashir, M., and Hampton, R. O. 1996. Detection and identification of seed-borne viruses from cowpea (Vigna unguiculata (L.) Walp.) germplasm. Plant Pathol. 45:54-58.

3. Doolittle, S. P., and Gilbert, W. W. 1919. Seed transmission of cucurbit mosaic by the wild cucumber. Phytopathology 9:326-327.

4. Gillaspie, A. G., Jr., Hopkins, M. S., Pinnow, D. L., and Hampton, R. O. 1995. Seedborne viruses in preintroduction seed lots and establishment of virus-free accessions. Plant Dis. 79:388-391.

5. Habili, N., and Francki, R. I. B. 1974. Com- parative studies on tomato aspermy and cucumber mosaic viruses. III. Further studies on relationship and construction of a virus from parts of the two viral genomes. Virology 61:443-449.

6. Hajimorad, M. R., Kurath, G., Randles, J. W., and Francki, R. I. B. 1991. Change in phenotype and encapsidated RNA segments of an isolate of alfalfa mosaic virus: An influence of host passage. J. Gen. Virol. 72:2885-2893.

7. Hu, C.-C., Aboul-Ata, A. E., Naidu, R. A. and Ghabrial, S. A. 1997. Evidence for the occurrence of two distinct subgroups of peanut stunt cucumovirus strains: Molecular characterization of RNA3. J. Gen. Virol. 78:929-939.

8. Jordan, R. L., and Dodds, J. A. 1985. Doublestranded RNA in detection of diseases of known and unproven viral etiology. Acta Hortic. 164:101-108.

9. Kroner, P., Richards, D., Traynor, P., and Ahlquist, P. 1989. Defined mutations in a small region of the brome mosaic virus $2 \mathrm{a}$ gene cause diverse temperature-sensitive RNA replication phenotypes. J. Virol. 63:5302-5309.

10. McGovern, R. J., Polston, J. E., Danyluk, G. M., Hiebert, E., Abouzid, A. M., and Stansly, P. A. 1994. Identification of a natural weed host of tomato mottle geminivirus in Florida. Plant Dis. 78:1102-1106.

11. Meiners, J. P., Waterworth, H. E., Smith, F. F., Alconero, R., and Lawson, R. H. 1977. A seed-transmitted strain of cucumber mosaic virus isolated from bean. J. Agric. Univ. Puerto Rico 61:137-147.

12. Mink, G. I. 1972. Peanut stunt virus. Commonw. Mycol. Inst./Assoc. Appl. Biol. Descriptions of Plant Viruses, No. 92.

13. Naidu, R. A., Collins, G. B., and Ghabrial, S A. 1991. Symptom-modulating properties of peanut stunt virus satellite RNA sequence variants. Mol. Plant-Microbe Interact. 4:268275.

14. Owen, J., and Palukaitis, P. 1988. Characterization of cucumber mosaic virus. 1. Molecular heterogeneity mapping of RNA3 in eight CMV strains. Virology 166:495-502.

15. Pio-Ribeiro, G. S., Wyatt, S. D., and Kuhn, C. W. 1978. Cowpea stunt: A disease caused by a synergistic interaction of two viruses. Phytopathology 68:1260-1265.

16. Rizzo, T. M., and Palukaitis, P. 1990. Construction of full-length clones of cucumber mosaic virus RNAs 1, 2, and 3: Generation of infectious RNA transcripts. Mol. Gen. Genet. 222:249-256

17. Roossinck, M. J., Kaplan, I., and Palukaitis, P. 1997. Support of cucumber mosaic virus satellite RNA maps to a single amino acid promixal to the helicase domain of the helper virus. J. Virol. 71:608-612.

18. Sambrook, J., Fritsch, E. F., and Maniatis, T. A. 1989. Molecular cloning: A laboratory manual. 2nd ed. Cold Spring Harbor Laboratory, Cold Spring Harbor, NY

19. Yarwood, C. E. 1979. Host passage effects with plant viruses. Adv. Virus Res. 25:169-190.

\section{ERRATUM / Volume 82, Number 4, 1998}

In the article "Characterization of a Severe Strain of Cucumber Mosaic Cucumovirus Seedborne in Cowpea" by A. G. Gillaspie, Jr., M. R. Hajimorad, and S. A. Ghabrial, on pages 419-422, Figure 1 should appear as follows:

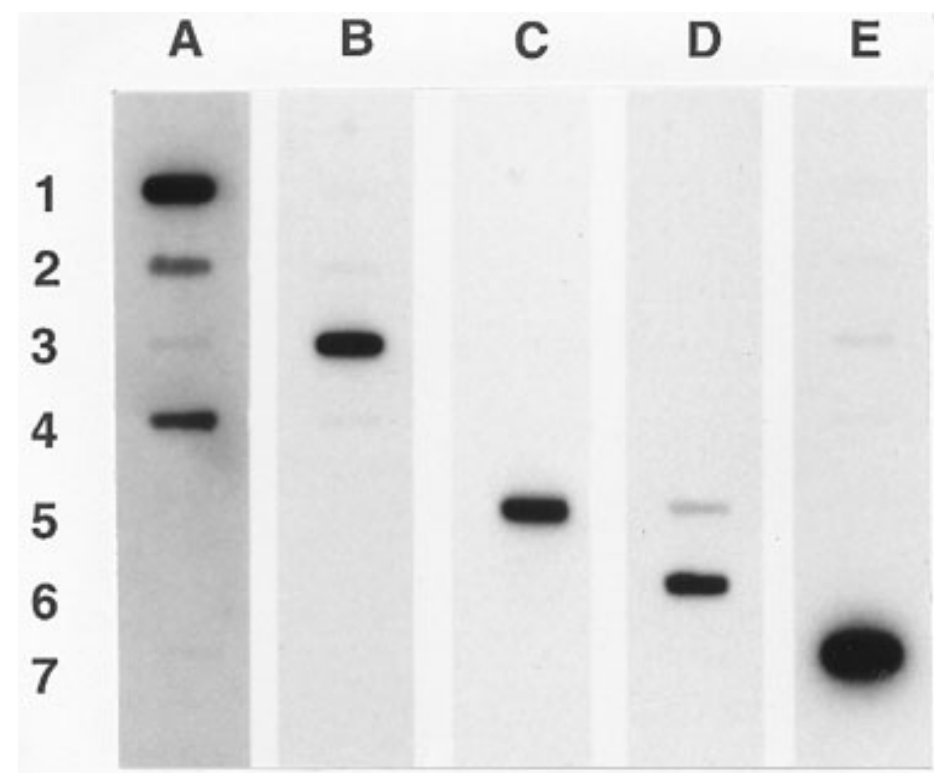

Fig. 1. Slot blot hybridization analysis of cucumovirus RNAs. Viral RNAs $(0.5 \mu \mathrm{g})$ in TE buffer isolated from purified virions of cucumber mosaic virus (CMV)-Fny (1), CMV-Csb isolate 276 (2), CMV-LS (3), CMV-Csb isolate 315 (4), peanut stunt virus (PSV)-ER (5), PSV-W (6), and tomato aspermy virus (7) were spotted onto Hybond $\mathrm{N}^{+}$membranes and hybridized ${ }^{32} \mathrm{P}$-labeled cDNA to genomic RNAs of CMV-Fny, CMV-LS, PSV-ER, PSV-W, and TAV-V, panels A to E, respectively. CMV-Csb isolates hybridized only with cDNA probes to CMV-Fny RNAs. 